\title{
北方农牧交错带温性盐碱化草地土壤呼吸对不同 形态氮添加和刈割的响应
}

\author{
胡姝娅 ${ }^{1,2}$ フ华杰 ${ }^{1,3}$ 王惠玲 $^{3}$ 薄元超 $^{3}$ 申 颜 ${ }^{1}$ 孙 伟 ${ }^{4}$ 董宽虎 $^{3}$ 黄建辉 $^{1,2}$ \\ 王常慧 ${ }^{*}$
}

${ }^{1}$ 中国科学院植物研究所植被与环境变化国家重点实验室, 北京 $100093 ;{ }^{2}$ 中国科学院大学, 北京 $100049 ;{ }^{3}$ 山西农业大学动物科技学院, 山西晋中 030801; ${ }^{4}$ 东北师范大学植被生态学教育部重点实验室, 长春 130024

摘 要 农牧交错带草地生态系统兼受农业和牧业的影响, 属于脆弱生态系统, 尤其是养分贫㾑的盐碱化草地, 其生态系统 结构和功能对外界干扰的响应更加强烈。位于晋西北地区的农牧交错带盐碱化草地, 地理位置独特, 区别于天然牧区草地生 态系统。由于毗邻农田, 农业氮肥的过量使用促进了活性氮气体排放, 同时使得农牧交错带草地土壤碳氮循环发生改变。刈 割是北方农牧交错草地生态系统的主要管理方式, 为了深入探究氮添加和刈割管理方式对农牧交错带草地碳循环的影响, 进 一步厘清该区域草地生态系统的碳动态问题, 该研究设置了一个不同形态氮添加和刈割的裂区实验, 测定土壤呼吸对不同形 态氮肥添加和则割的响应, 为进一步科学管理该区域草地提供可靠的依据。实验样地位于山西省右玉县境内的“山西农业大 学农牧交错带草地生态系统野外观测研究站”, 于2017年设置不同形态氮添加和刈割处理, 实验处理包括对照(不刈割和刈 割)、尿素添加、缓释尿素添加、刈割+尿素添加、刈割+缓释尿素添加, 每种处理 6 个重复, 共36个小区。在不同处理条件下 测定土壤呼吸速率、土壤温度、土壤水分、土壤微生物生物量、土壤无机氮含量、植物地上和地下生物量, 并计算土壤累积 碳排放量及 $\mathrm{CO}_{2}$ 通量。研究结果表明: (1)短期(2017-2018年)尿素和缓释尿素的添加显著提高了该地区土壤呼吸速率和土壤累 积碳排放量。与添加缓释尿素相比, 添加尿素处理下的土壤呼吸速率和累积碳排放量更高; (2)刚割显著降低土壤呼吸速率和 累积碳排放量; (3)短期氮添加和则割的交互作用对土壤呼吸速率没有显著影响。因此, 短期氮添加促进了北方农牧交错带盐 碱化草地土壤碳释放, 刈割抑制土壤呼吸, 降低了累积碳排放量, 这可能是由于刈割移除地上植物, 减少了调落物的输入, 底物减少导致土壤微生物活性降低。但是随着处理时间的延长, 氮添加和刈割对该农牧交错带盐碱化草地土壤碳动态的影响 还有待进一步探究和发现。

关键词 刈割; 氮形态; 土壤呼吸; 农牧交错带; 盐碱化草地

胡姝娅, 习华杰, 王惠玲, 薄元超, 申颜, 孙伟, 董宽虎, 黄建辉, 王常慧 (2020). 北方农牧交错带温性盐碱化草地土壤呼吸对不同形态氮添加和刈割 的响应. 植物生态学报, 44, 70-79. DOI: 10.17521/cjpe.2019.0270

\section{Response of soil respiration to addition of different forms of nitrogen and mowing in a saline-alkali grassland in the northern agro-pastoral ecotone}

HU Shu-Ya ${ }^{1,2}$, DIAO Hua-Jie ${ }^{1,3}$, WANG Hui-Ling ${ }^{3}$, BO Yuan-Chao ${ }^{3}$, SHEN Yan ${ }^{1}$, SUN $\mathrm{Wei}^{4}$, DONG Kuan-Hu ${ }^{3}$, HUANG Jian-Hui ${ }^{1,2}$, and WANG Chang-Hui ${ }^{*}$

${ }^{1}$ State Key Laboratory of Vegetation and Environmental Change, Institute of Botany, Chinese Academy of Sciences, Beijing 100093, China; ${ }^{2}$ University of Chinese Academy of Sciences, Beijing 100049, China; ${ }^{3}$ College of Animal Science and Veterinary Medicine, Shanxi Agricultural University, Jinzhong, Shanxi 030801, China; and ${ }^{4}$ Key Laboratory of Vegetation Ecology, Ministry of Education, Northeast Normal University, Changchun 130024, China

\footnotetext{
Abstract

Aims The agro-pastoral ecotone is considered as fragile ecosystems which are strongly affected by agriculture and animal husbandry. The saline-alkali grassland is a unique grassland type in the agro-pastoral ecotone. A large amount of fertilizers are used to increase productivity in this area, which also promotes the emission of reactive nitrogen $(\mathrm{N})$ gases and leads to the changes in soil carbon and $\mathrm{N}$ cycles. Mowing is a primary management practice in the agro-pastoral grassland in northern China. In order to explore the impact of $\mathrm{N}$ addition and mowing

收稿日期Received: 2019-10-15 接受日期Accepted: 2020-01-05

基金项目：国家自然科学基金项目(31770526和31872406)和国家重点研发计划(2017YFA0604802和2016YFC0500703)。Supported by the National Natural Science Foundation of China (31770526 and 31872406), and the National Key R\&D Program of China (2017YFA0604802 and 2016YFC0500703).

* 通信作者Corresponding author (wangch@ibcas.ac.cn)
} 
on carbon dynamic in this saline-alkali grassland located in the agro-pastoral ecotone, we determined the response of soil respiration to $\mathrm{N}$ addition and mowing.

Methods This study area is located in Youyu County, an agro-pastoral grassland ecosystem in northern China. The field experiment was set up in May, 2017. The treatments included: control (without mowing and mowing), addition of urea, addition of slow release urea, addition of urea + mowing, addition of slow release urea + mowing. Each treatment included 6 replicates. Therefore, there were totally 36 plots in this experiment. Soil respiration rate, soil temperature, soil moisture content, microbial biomass, inorganic $\mathrm{N}$ content, above-ground and below-ground biomass were measured under different treatments, and the cumulative carbon emissions and $\mathrm{CO}_{2}$ fluxes were calculated.

Important findings Our results showed that: (1) Short-term (2017-2018) N addition significantly increased soil respiration rates and soil cumulative carbon emissions. Meanwhile, soil respiration rates and cumulative carbon emissions were significantly higher under urea treatment than those under slow release urea addition. (2) Mowing significantly reduced soil respiration rates and cumulative carbon emissions. (3) The interaction of short-term $\mathrm{N}$ addition and mowing had no significant effect on soil respiration rate. Therefore, short-term $\mathrm{N}$ addition can promote soil carbon release from the saline-alkali grassland in the agro-pastoral ecotone of northern China. Mowing can reduce soil respiration and decrease cumulative of carbon emissions. This may be because that mowing reduced the input of litter and further reduced soil substrate for microbes, which led to a decrease in soil microbial activity. However, long-term effect of $\mathrm{N}$ addition and mowing on soil carbon dynamics in saline-alkaline grasslands in the agro-pastoral ecotone still needs to be further explored.

Key words mowing; nitrogen forms; soil respiration; agro-pastoral ecotone; saline-alkali grassland

Hu SY, Diao HJ, Wang HL, Bo YC, Shen Y, Sun W, Dong KH, Huang JH, Wang CH (2020). Response of soil respiration to addition of different forms of nitrogen and mowing in a saline-alkali grassland in the northern agro-pastoral ecotone. Chinese Journal of Plant Ecology, 44, 70-79. DOI: 10.17521/cjpe.2019.0270

土壤碳(C)库是陆地生态系统最大的碳库, 储 存了大约 $1500 \mathrm{Pg}$ 的有机碳。土壤呼吸所释放的 $\mathrm{CO}_{2}$ 是土壤与大气之间最大的气体交换通量之一, 因此 土壤呼吸微小的变化都会对大气 $\mathrm{CO}_{2}$ 浓度及土壤碳 库产生巨大的影响(Schlesinger \& Andrews, 2000; Lal, 2004)。草地生态系统有机碳储量约占陆地总碳 储量的 $15 \%$, 是全球碳库的重要组成部分(赵同谦等, 2004)。人类活动如施肥、刈割、放牧等是导致草地 土壤碳排放变化的主导因子(张英俊等, 2013)。

草地外源氮 $(\mathrm{N})$ 输入不仅会直接影响土壤呼吸 速率的大小, 还会通过改变植被组成、根系生长、 土壤微生物活性(Peng et al., 2017)以及土壤微环境 等因子直接或间接影响土壤呼吸和土壤碳排放(贺 云龙等, 2018)。研究发现土壤呼吸受草地类型、施 氮水平、施氮年限、施氮频次等方面的影响(王丹和 陈永金, 2015; Peng et al., 2017; 胡伟等, 2019; 康静 等, 2019)。但是由于氮添加时间、氮添加年限、氮 的形态以及添加氮的水平在不同的控制实验存在差 异, 因此草地土壤呼吸速率对氮添加的响应尚无定 论, 基于上述处理的不同, 现有的研究发现氮添加 对土壤呼吸的影响有促进(Peng et al., 2011; 张蓄等, 2017), 无影响(Liu \& Greaver, 2010; Qi et al., 2014)
和抑制(Li et al., 2018a; Wei et al., 2018)作用等。对 于草地利用方式而言, 刈割对不同类型草地以及相 同类型下不同利用方式草地的土壤呼吸都会产生差 异性影响(孙振中等, 2012; 马俐等, 2016)。

我国北方农牧交错带草地面积约为 $5 \times 10^{5} \mathrm{~km}^{2}$ (何立环等, 2014), 是草地的重要组成部分。与天然 牧区的草地不同, 农牧交错带草地生态系统更多地 受到农业管理方式和管理制度的影响(如施肥、刈 割)(李博, 1997; 王林峰等, 2018)。盐碱化草地广泛 分布于全球各地, 具有巨大的固碳潜力(谢静霞等, 2008; 王新源等, 2012; 米迎宾等, 2016)。我国盐碱 化草地主要分布在北方地区且随着畜牧业的发展盐 碱化日益严重(Zhang et al., 2016)。由于盐碱草地研 究的缺乏, 目前农牧交错带盐碱地草地生态系统土 壤呼吸对不同管理利用方式(施氮、刈割)的响应尚 不明确。因此, 本研究旨在利用中国北方农牧交错 带盐碱地草地生态系统的氮添加和划割处理控制试 验平台，分析探讨不同形态氮添加和刚割对北方农 牧交错带盐碱地草地生态系统土壤呼吸的影响, 揭 示影响土壤呼吸的主控因子, 从土壤碳排放的角 度为该地区草地管理提供数据支持和科学的管理 依据。 


\section{1 材料和方法}

\section{1 研究地概况}

本研究样地位于山西省右玉县山西农业大学农 牧交错带草地生态系统野外观测研究站 $\left(39.28^{\circ} \mathrm{N}\right.$, $112.29^{\circ} \mathrm{E}$ ), 属温带大陆性季风气候, 年平均气温 $4.7{ }^{\circ} \mathrm{C}$, 生长季平均气温 $16.7{ }^{\circ} \mathrm{C}$, 全年月平均最高气 温 $22.4{ }^{\circ} \mathrm{C}$, 月平均最低气温 $-17{ }^{\circ} \mathrm{C}$ 。海拔 $1348 \mathrm{~m}$, 年降水量为 $435 \mathrm{~mm}$, 无霜期 $100-120$ 天, 年日照率为 $30 \%$, 土壤 $\mathrm{pH}$ 约为 8.7 , 属于中度盐碱化草地。群落优 势种是羊草(Leymus chinensis)、赖草(L. secalinus)、克 氏针茅(Stipa capillata)等, 植被类型接近草甸。

\section{2 实验设计}

本实验采用完全随机区组设计, 实验设置对 照、尿素和缓释尿素两种形态的氮添加处理, 施氮 量为 $10 \mathrm{~g} \mathrm{~N} \cdot \mathrm{m}^{-2} \cdot \mathrm{a}^{-1}$ 。在不同形态的氮添加处理下设 置了刚割和不刚割两种方式, 实验一共设置6种处 理, 分别为对照处理、刈割处理、添加尿素处理、 添加缓释尿素处理、尿素+刚割处理和缓释尿素+刈 割处理。每个处理设置 6 个重复, 共计 36 个样方。样 方大小为 $8 \mathrm{~m} \times 9 \mathrm{~m}$, 样方间设置 $2 \mathrm{~m}$ 的缓冲带。实验 从2017年5月开始, 氮添加时间为每年5月初降雨前 一次性施入, 添加方式为撒施, 刈割时间为每年 8 月 中上旬。对照处理不采取任何措施。

\section{3 土壤呼吸的测定和累积排放量的计算}

在每个样方内插入 2 个PVC环(直径 $10 \mathrm{~cm}$, 高 $6 \mathrm{~cm}$ ), 环口距离地面约 $3 \mathrm{~cm}$ 。运用LI-COR840便携 式土壤 $\mathrm{CO}_{2}$ 通量系统(Lincoln, NE, USA)测定土壤呼 吸, 测定时间选在生长季(6-9月)天气晴朗的上午, 每个月分别于月初和月中测定2次。2017年共测定9 次(8月测定3次), 2018年共测定8次。

土壤 $\mathrm{CO}_{2}$ 累积排放量计算公式为:

$$
M=\sum\left(F_{i+1}+F_{i}\right) / 2 \times\left(t_{i+1}-t_{i}\right) \times 24
$$

式中, $M$ 为 $\mathrm{CO}_{2}$ 排放累积值 $\left(\mathrm{mg} \cdot \mathrm{m}^{-2}\right), F$ 为 $\mathrm{CO}_{2}$ 排放速率 $\left(\mathrm{mg} \cdot \mathrm{m}^{-2} \cdot \mathrm{d}^{-1}\right), t$ 为采集时间, $i$ 表示每年的第 $i$ 次取样。

1.4 地上生物量(AGB)和地下生物量(BGB)的测定

于 2017 和 2018 年 8 月中旬, 在每个样方内随机 选取 $2 \mathrm{~m} \times 0.2 \mathrm{~m}$ 的两个样方, 分别获取植物地上 生物量。将收获的生物量样品放入烘箱, $65{ }^{\circ} \mathrm{C}$ 烘至 恒质量(约 $48 \mathrm{~h}$ )后称质量, 计算地上生物量。在收获 完地上生物量的样方内, 用内径为 $7.5 \mathrm{~cm}$ 的土壤根 钻分别钻取 0-10 $\mathrm{cm} 、 10-20 \mathrm{~cm}$ 和 $20-30 \mathrm{~cm}$ 的土层土
样。土样过 $2 \mathrm{~mm}$ 篎，收集根系，将根系洗净后放入 烘箱, 于 $65{ }^{\circ} \mathrm{C}$ 烘至恒质量(约48 h) 后称质量, 计算 地下生物量。

\section{5 土壤温度、含水量、微生物生物量以及其他土 壤理化性质的测定}

土壤温度和土壤含水量的测定与土壤呼吸的测 定同时进行, 将LI-COR840的温度探针插在PVC环 附近测定 0-10 cm土层的土壤温度。用TDR-200探测 器(Spectrum Technologies, Plainfield, USA)在相同深 度测定土壤体积含水量。

于2017和2018年的 8 月采集土壤表层 $(0-10 \mathrm{~cm})$ 样品。采集的土样一部分风干后, 用电位法测定土 壤pH值。用N/C 3100 TOC/TN分析仪(Analytik Jena AG, Jena, Germany)测定土壤总有机碳(TOC)和总氮 (TN) 含量。新鲜土样用氯仿熏蒸法测定土壤微生物 生物量碳氮(Vance et al., 1987)。土壤可溶性有机碳 (DOC) 含量采用硫酸钾浸提法测定, 另取部分新鲜 土样过 $2 \mathrm{~mm}$ 笁, 经 $0.5 \mathrm{~mol} \cdot \mathrm{L}^{-1}$ 的 $\mathrm{K}_{2} \mathrm{SO}_{4}$ 溶液浸提后, 用流动分析仪(FIAstar 5000 Analyzer, Foss Tecator, Denmark)测定铵态氮和硝态氮含量。

\section{6 数据分析}

用重复测定方差分析法和多因素方差分析对土 壤呼吸速率、土壤含水量、土壤温度等指标进行分 析。采用线性混合模型明确氮添加和刈割对土壤呼 吸累积量的影响。采用一般线性回归法和Pearson相 关性系数评价土壤呼吸与土壤温度、含水量以及土 壤无机氮、微生物生物量碳、氮和地上生物量、地 下生物量等因子之间的相关性。响应比计算公式为: (处理后的土壤呼吸速率-对照组的土壤呼吸速率)/ 对照组的土壤呼吸速率 $\times 100 \%$ 。数据分析使用SPSS 22.0 (显著性水平 $p=0.05$ ), 使用Sigmaplot 12.5 作图。

\section{2 结果}

\section{1 土壤温度水分变化}

2017年生长季(6-9月)土壤温度的变化范围为 11.97-26.07 ${ }^{\circ} \mathrm{C}$, 其中最高气温出现在7月, 最低气 温出现在9月。2018年6-9月间土壤温度在16.97$25.78{ }^{\circ} \mathrm{C}$ 之间, 其中最高值出现在 8 月, 最低值出现 在9月。2017年各处理间和2018年各处理间土壤温度 与对照相比均无显著差异 $(p>0.05)$ (图1)。2017年 6-9月土壤体积含水量月平均值在7.78-29.64 $\mathrm{cm} \cdot \mathrm{cm}^{-3}$ 之间, 其中最大值出现在6月, 最小值出现在7月。 


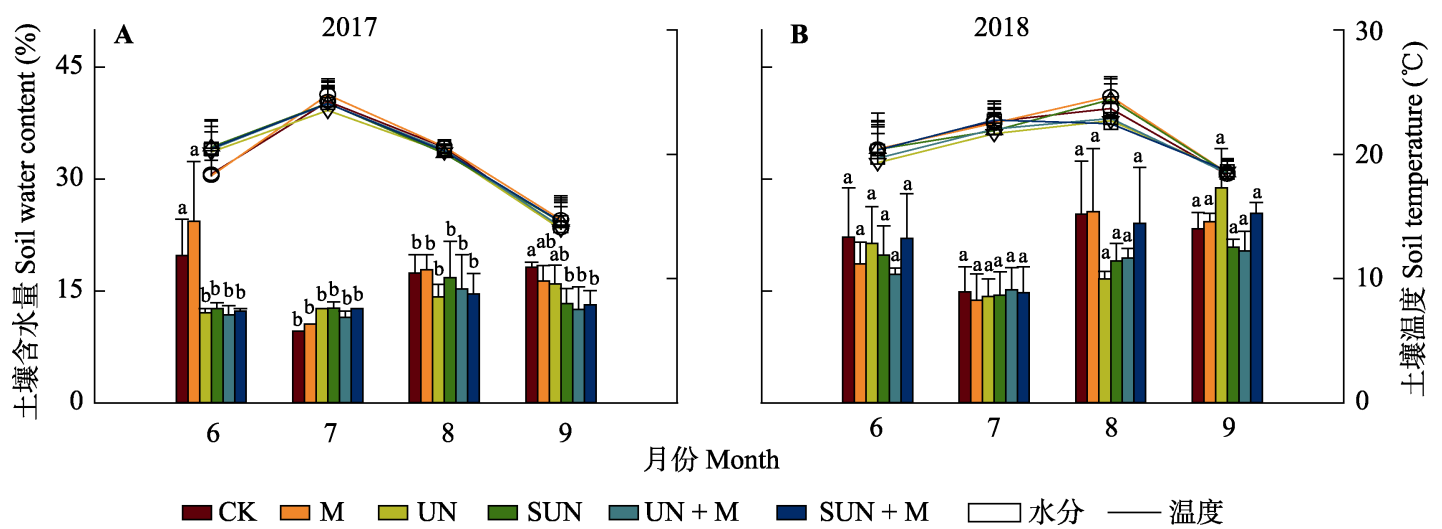

图12017和2018年各处理间土壤含水量及土壤温度的季节动态(平均值+标准误差)。CK, 对照处理; M, 刈割处理; SUN, 添加缓释尿素处理; SUN + M, 添加缓释尿素+刈割处理; UN, 添加尿素处理; UN + M, 添加尿素+刈割处理。不同小写字母 表示差异显著 $(p<0.05)$ 。

Fig. 1 Seasonal dynamics of soil water content and soil temperature in 2017 and 2018 (mean + SE). CK, control treatment; M, mowing treatment; SUN, slow release urea treatment; SUN + M, slow release urea and mowing treatment; UN, urea treatment; $\mathrm{UN}+\mathrm{M}$, urea and mowing treatment. Different lowercase letters indicate significant differences $(p<0.05)$.

2018 年土壤体积含水量月平均值在 10.28-29.17 $\mathrm{cm} \cdot \mathrm{cm}^{-3}$ 之间, 最大值出现在 8 月, 最小值出现在 7 月。2017年6月，与对照相比施氮处理和施氮+刈割 处理后土壤含水量显著降低 $(p<0.05), 9$ 月施加缓释 尿素、尿素+刈割、缓释尿素+刈割处理下土壤含水 量显著低于对照 $(p<0.05)$ 。2018年6-9月各处理下土 壤含水量与对照相比无显著差异 $(p>0.05)$ (图1)。

\section{2 不同处理下土壤呼吸速率的生长季动态}

土壤呼吸在氮添加的前两年(2017和2018年)测 定时间内均呈现出明显的季节动态。2017年生长季
各处理下的土壤呼吸与对照表现出相似的波动。总 体来看, 生长季旺期(7月及 8 月)大于生长季初期 $(6$ 月)和末期(9月), 土壤呼吸最小值出现在6月，最大 值出现在7月。2018年7月刈割处理, 尿素+刈割处理和 缓释尿素 + 刈割处理下土壤呼吸速率分别比对照显 著降低了 $78 \%$ 、42\%和 $65 \%$ ，8月刈割处理，尿素+刈 割处理和缓释尿素+刚割处理下土壤呼吸速率分别 比对照显著降低了 54\%、38\%和43\% $(p<0.05)$ (图 $2 \mathrm{~A} 、 2 \mathrm{~B})$ 。多因素方差分析结果表明，测定时间对土 壤呼吸速率的影响达到极显著水平 $(p<0.01)$ (表1)。

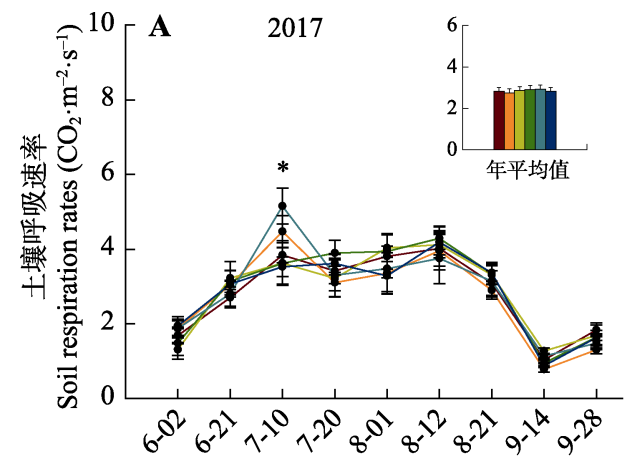

日期 Date

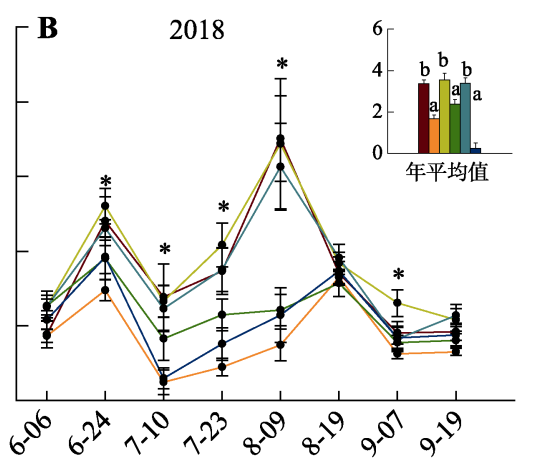

日期 Date

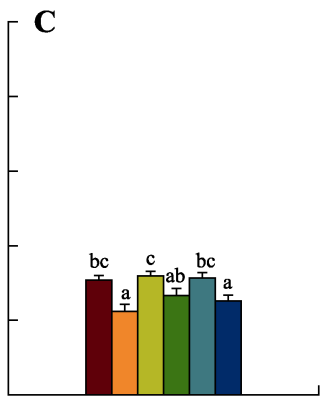

两年平均值 Two-year mean value

图2 北方农牧交错带温性盐碱化草地不同处理的土壤呼吸速率季节动态(平均值+标准误差)。CK, 对照处理; M, 刏割处 理; SUN, 添加缓释尿素处理; SUN + M, 添加缓释尿素+刈割处理; UN, 添加尿素处理; UN + M, 添加尿素+刈割处理。不 同小写字母表示差异显著 $(p<0.05)$ 。

Fig. 2 Seasonal dynamics of soil respiration rates under different treatments of saline-alkali grassland in the northern agricultural-pastoral ecotone (mean $+S E$ ). CK, control treatment; M, mowing treatment; SUN, slow release urea treatment; SUN + M, slow release urea and mowing treatment; $\mathrm{UN}$, urea treatment; $\mathrm{UN}+\mathrm{M}$, urea and mowing treatment. Different lowercase letters indicate significant differences $(p<0.05)$. 
表1＼cjkstart氮添加、刈割及其交互作用对北方农牧交错带温性盐碱化草地土 壤呼吸的影响

Table 1 Effects of $\mathrm{N}$ addition, mowing and their interactions on soil respiration in saline-alkali grassland in the northern agricultural-pastoral ecotone

\begin{tabular}{lrrc}
\hline 因素 Factor & $d f$ & \multicolumn{1}{c}{$F$} & $P$ \\
\hline 测定时间 Measuring time (Time) & 16 & 35.947 & 0.000 \\
氮添加 Nitrogen addition (N) & 2 & 3.955 & 0.020 \\
刈割 Mowing & 1 & 55.789 & 0.000 \\
测定时间×氮添加 $($ Time $\times \mathrm{N})$ & 32 & 0.547 & 0.981 \\
测定时间 $\times$ 刈割 $($ Time $\times$ Mowing) & 16 & 11.760 & 0.000 \\
施氮处理 $\times$ 刈割 $(\mathrm{N} \times$ Mowing) & 2 & 0.150 & 0.481 \\
测定时间×氮添加 $\times$ 刈割 $($ Time $\times \mathrm{N} \times$ Mowing) & 32 & 0.579 & 0.977
\end{tabular}

*, 相关性在 0.05 水平上显著; $* *$, 相关性在 0.01 水平上显著。

*, correlation is significant at the 0.05 level; **, correlation is significant at the 0.01 level.

从每年的生长季平均值上看, 2017年生长季不同 处理下土壤呼吸速率的大小分别是 $(2.83 \pm 0.36) \mu \mathrm{mol}$ $\mathrm{CO}_{2} \cdot \mathrm{m}^{-2} \cdot \mathrm{s}^{-1}(\mathrm{CK}),(2.87 \pm 0.36) \mu \mathrm{mol} \mathrm{CO} \mathrm{CO}_{2} \cdot \mathrm{m}^{-2} \cdot \mathrm{s}^{-1}$ (UN), $(2.87 \pm 0.43) \mu \mathrm{mol} \mathrm{CO} \mathrm{CO}^{-2} \cdot \mathrm{s}^{-1}$ (SUN), $(2.76 \pm$ $0.40) \mu \mathrm{mol} \mathrm{CO} \mathrm{CO}_{2} \cdot \mathrm{m}^{-2} \cdot \mathrm{s}^{-1}(\mathrm{CK}+\mathrm{M}),(2.91 \pm 0.42) \mu \mathrm{mol}$ $\mathrm{CO}_{2} \cdot \mathrm{m}^{-2} \cdot \mathrm{s}^{-1}(\mathrm{UN}+\mathrm{M}),(2.84 \pm 0.36) \mu \mathrm{mol}$ $\mathrm{CO}_{2} \cdot \mathrm{m}^{-2} \cdot \mathrm{s}^{-1}(\mathrm{SUN}+\mathrm{M})$, 但是, 这些处理间土壤呼 吸速率没有显著差异 $(p>0.05)$ 。2018年生长季不同 处理下土壤呼吸速率的大小分别是(3.36 \pm 0.32$) \mu \mathrm{mol}$ $\mathrm{CO}_{2} \cdot \mathrm{m}^{-2} \cdot \mathrm{s}^{-1}(\mathrm{CK}),(3.73 \pm 0.32) \mu \mathrm{mol} \mathrm{CO} \mathrm{CO}_{2} \cdot \mathrm{m}^{-2} \cdot \mathrm{s}^{-1}$ (UN), $(3.39 \pm 0.28) \mu \mathrm{mol} \mathrm{CO} \mathrm{CO}^{-2} \cdot \mathrm{s}^{-1}$ (SUN), $(2.16 \pm$ $0.37) \mu \mathrm{mol} \mathrm{CO} \cdot \mathrm{m}^{-2} \cdot \mathrm{s}^{-1}(\mathrm{CK}+\mathrm{M}),(2.38 \pm 0.17) \mu \mathrm{mol}$ $\mathrm{CO}_{2} \cdot \mathrm{m}^{-2} \cdot \mathrm{s}^{-1}(\mathrm{UN}+\mathrm{M}),(2.20 \pm 0.20) \mu \mathrm{mol}$ $\mathrm{CO}_{2} \cdot \mathrm{m}^{-2} \cdot \mathrm{s}^{-1}(\mathrm{SUN}+\mathrm{M})$ ，其中 $\mathrm{M} 、 \mathrm{UN}+\mathrm{M} 、 \mathrm{SUN}+\mathrm{M}$ 处理下土壤呼吸速率显著低于 $\mathrm{CK}$ 处理 $(p<0.05)$ (图 $2 \mathrm{~A} 、 2 \mathrm{~B})$, 从两年平均值上看, $\mathrm{M} 、 \mathrm{UN}+\mathrm{M}$ 和 $\mathrm{SUN}+$ $\mathrm{M}$ 处理下土壤呼吸速率显著降低 $(p<0.05)$ 。各处理 对土壤呼吸速率的影响从大到小依次是UN $>\mathrm{SUN}$ $>\mathrm{CK}>(\mathrm{UN}+\mathrm{M})>(\mathrm{SUN}+\mathrm{M})>\mathrm{M}($ 图2C)。

2.3 短期氮添加、刈割及二者交互作用对土壤呼吸 速率及土壤累积碳排放量的影响

方差分析结果表明, 氮添加处理显著提高了土 壤呼吸速率和土壤累积碳排放量 $(p<0.05)$ (表 1 , 表 2), 2017年尿素添加处理下的土壤呼吸速率响应比 和缓释尿素添加处理下的土壤呼吸速率响应比均为 正值, 两种氮添加类型之间的响应比无显著差异 $(p$ $>0.05) ; 2018$ 年添加尿素处理下土壤呼吸响应比为 正值, 添加缓释尿素处理下土壤呼吸的响应比为负 值, 二者差异达到显著水平 $(p<0.05)$ (图3)。从累积 碳排放量上看, 2017年氮添加处理下土壤累积碳排 放量与对照相比无明显变化, 2018年氮添加处理下
表2 氮添加、刈割及其交互作用对年平均土壤累积碳排放量的影响 Table 2 Effects of $\mathrm{N}$ addition, mowing and their interactions on annual cumulative carbon emissions.

\begin{tabular}{lccc}
\hline & $d f$ & $F$ & $P$ \\
\hline 固定效应 Fixed effects & 2 & 6.751 & 0.005 \\
氮添加 Nitrogen addition (N) & 1 & 0.124 & 0.728 \\
刈割 Mowing & 2 & 0.239 & 0.789 \\
氮添加×刈割 $(\mathrm{N} \times$ Mowing) & & & \\
随机效应 Random effects & - & 1.313 & 0.189 \\
小区 Block & & & \\
\hline
\end{tabular}

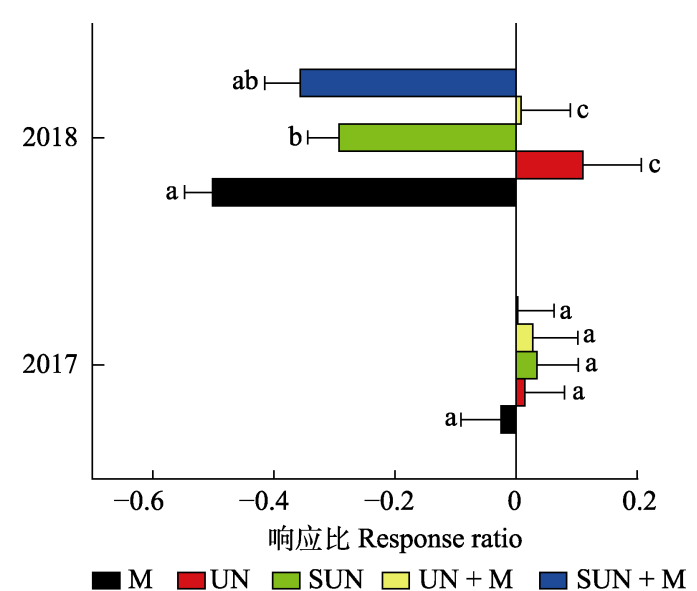

图3土壤呼吸对不同处理的响应(平均值+标准误差)。 $\mathrm{M}$, 刚割处理; SUN, 添加缓释尿素处理; SUN + M, 添加缓释 尿素+刚割处理; UN, 添加尿素处理; UN + M, 添加尿素+ 刏割处理。不同小写字母表示差异显著 $(p<0.05)$ 。

Fig. 3 Response of soil respiration to different treatments $($ mean $+S E)$. M, mowing treatment; SUN, slow release urea treatment; SUN $+\mathrm{M}$, slow release urea and mowing treatment; $\mathrm{UN}$, urea treatment; $\mathrm{UN}+\mathrm{M}$, urea and mowing treatment. Different lowercase letters indicate significant differences $(p<0.05)$.

土壤累积碳排放量显著提高, 添加尿素处理下土壤 碳累积排放量高于添加缓释尿素 $(p<0.05)$ (图4A、

$4 \mathrm{~B})$ 。从两年生长季碳排放量平均值上看, 各处理间 无显著差异(图4C)。

刚割处理显著降低了土壤呼吸速率 $(p<0.01)$ (表1)。2017和2018年刈割处理对土壤呼吸的影响均 表现为负效应，2017年土壤呼吸速率对刈割处理的 响应比与其他处理的响应比相比差异不显著。2018 年刈割处理下土壤呼吸的响应比显著低于添加尿素 处理、添加缓释尿素处理和添加尿素+刈割处理(图 3)。从累积排放量上看, 与对照相比, 2017年刈割处 理下土壤 $\mathrm{CO}_{2}$ 累积排放量无明显变化(图4A); 2018 年刈割处理下土壤 $\mathrm{CO}_{2}$ 累积排放量显著降低 $(p<$ 0.05)(图4B); 从两年生长季排放量均值上看, 刈割 处理与对照相比无显著差异(图4C)。 


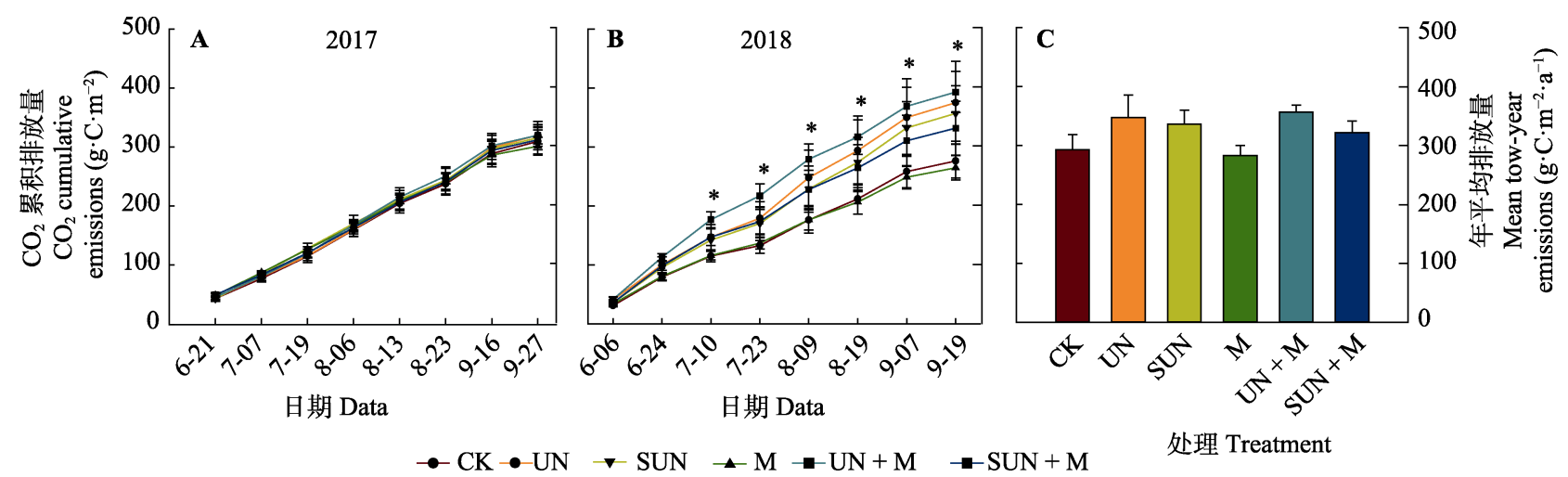

图4 北方农牧交错带温性盐碱化草地不同处理的生长季土壤 $\mathrm{CO}_{2}$ 排放量(平均值+标准误差)。 $\mathrm{CK}$, 对照处理; $\mathrm{M}$, 刏割处理; SUN, 添加缓释尿素处理; SUN + M, 添加缓释尿素+刈割处理; UN, 添加尿素处理; UN + M, 添加尿素+刈割处理。*, 差异 性在 0.05 水平上显著。

Fig. $4 \quad \mathrm{CO}_{2}$ cumulative emissions during the growing season under different treatments of saline-alkali grassland in the northern agricultural-pastoral ecotone (mean $+S E$ ). CK, control treatment; M, mowing treatment; SUN, slow release urea treatment; SUN $+\mathrm{M}$, slow release urea and mowing treatment; $\mathrm{UN}$, urea treatment; $\mathrm{UN}+\mathrm{M}$, urea and mowing treatment. *, difference is significant at the 0.05 level.

添加尿素 + 刈割处理和添加缓释尿素 + 刈割处 理下土壤呼吸速率低于对照处理(图2A、2B), 方差 分析结果表明, 氮添加和刈割的交互作用对土壤呼 吸速率的影响达到极显著水平 $(p<0.01)$ (表1)。2017 年添加尿素 + 刈割处理和添加缓释尿素 + 刈割处理 的土壤呼吸速率响应比为正值, 与对照相比差异不 显著。2018年添加尿素+刈割处理土壤呼吸速率响应 比为正值, 显著高于对照处理 $(p<0.05)$ (图3)。从累 积碳排放量上看, 2017年添加尿素+刈割处理和添 加缓释尿素+刚割处理下土壤累积碳排放量与对照 处理相比无明显变化; 2018年添加尿素+刈割处理和 添加缓释尿素 + 刈割处理下累积碳排放量显著低于 对照处理 $(p<0.05)$, 其中添加尿素+刈割处理的累积 碳排放量小于添加缓释尿素+刈割处理(图4A、4B)。 从生长季累积碳排放量上看, 施氮 + 刈割处理与对 照相比无显著差异(图4C)。

\section{4 土壤呼吸的影响因子}

相关分析结果表明, 土壤呼吸速率与铵态氮 $\left(\mathrm{NH}_{4}^{+}\right)$, 硝态氮 $\left.\left(\mathrm{NO}_{3}\right)^{-}\right)$含量呈显著负相关关系 $(p<$ $0.05)$, 与上壤微生物生物量碳( $\mathrm{MBC}$ ) 呈显著正相关 关系 $(p<0.05)$ 。土壤呼吸速率与土壤温度在氮添加 处理、刈割处理和氮添加 + 刈割处理下都呈现出极 显著的线性正相关关系 $(p<0.01)$, 即土壤呼吸速率 随土壤温度的增加而升高。不同处理下土壤温度与 土壤呼吸速率的相关性均高于对照处理, 不同处理 下土壤温度对土壤呼吸速率的解释度分别为 $25 \%$ (CK)、39\% (刚割)、26\% (添加尿素)、26\% (添加缓
释尿素)、 $50 \%$ (添加尿素+刚割)、32\% (添加缓释尿 素+刚割)。土壤水分含量与土壤呼吸速率的相关性 不显著 $(p>0.05)$ (图5)。

\section{3 讨论}

\section{1 土壤呼吸对短期氮添加的响应}

短期不同形态氮添加提高了北方农牧交错带盐 碱草地的土壤呼吸速率, 与其他学者在紫花苜宿 (Medicago sativa)草地以及羊草草地中不同水平氮 添加的研究结果(孙海燕等, 2018; 胡伟等, 2019) 一 致。2018年土壤呼吸速率对氮添加的响应大于 2017 年, 这可能是由于氮添加对土壤呼吸的影响存在累 积效应(Du et al., 2018), 土壤呼吸对氮添加的响应 依赖于氮添加的持续时间, 会随处理持续时间的增 加而增强(Li et al., 2018b)。也有研究结果发现氮添 加会降低荒漠草原土壤呼吸速率, 这是由于土壤中 氮浓度的增加引起土壤酸化, 降低微生物生物量 (Ye et al., 2018)、微生物活性和酶活性, 抑制了土壤 微生物的呼吸(康静等, 2019)。我们研究地点的土壤 类型属于盐碱化草地, 氮添加两年后土壤 $\mathrm{pH}$ 并未显 著降低 $(p>0.05)$, 这表明盐碱草地短期氮添加不会 导致土壤呼吸速率受土壤酸化的影响而降低。施氮 会增加土壤中有效氮含量(Kang et al., 2016), 有效 氮含量的增加促进了植被生长, 使地下生物量增加 (Li et al., 2018b), 提高土壤自养呼吸(Chen et al., 2016; Li et al., 2018b); 土壤有效氮含量的增加还会 改变土壤微生物群落结构(Zhou et al., 2017), 增加 
土壤微生物生物量和微生物活性 (Zhang et al., 2017a), 提高土壤异养呼吸。本研究结果发现氮添 加显著提高土壤有效氮含量; 2018年氮添加处理下 土壤无机氮 $\left(\mathrm{NH}_{4}-\mathrm{N} 、 \mathrm{NO}_{3}-\mathrm{N}\right)$ 含量比2017年氮添加处 理下增加 $25 \%(p<0.01)$, 土壤呼吸速率与微生物生 物量碳显著正相关(表3)。土壤有效氮的增加促进土 壤微生物生长, 提高微生物代谢效率, 从而提高土 壤呼吸速率。土壤呼吸速率与无机氮呈负相关关系 可能是由于氮添加导致土壤氮有效性增加, 地上地 下生物量比值显著增加 $(p<0.01)$, 植物对地下部分
分配比例降低，自养呼吸速率降低而产生的。

本研究还发现, 尽管添加尿素和缓释尿素都促 进了土壤呼吸, 但两种氮肥对土壤呼吸的促进能力 不同, 添加尿素对土壤呼吸的促进作用大于添加缓 释尿素。由于尿素的释放速率大于缓释尿素, 尿素 一次性释放到土壤中, 不能立即被植物全部吸收利 用, 大量尿素留在土壤表面或变成气体挥发掉(陈 德明等, 2002), 并且在生长季施加氮肥, 较高的土 壤温度导致尿素加速挥发释放 $\mathrm{CO}_{2}$, 因此添加尿素 土壤 $\mathrm{CO}_{2}$ 排放量大于添加缓释尿素。
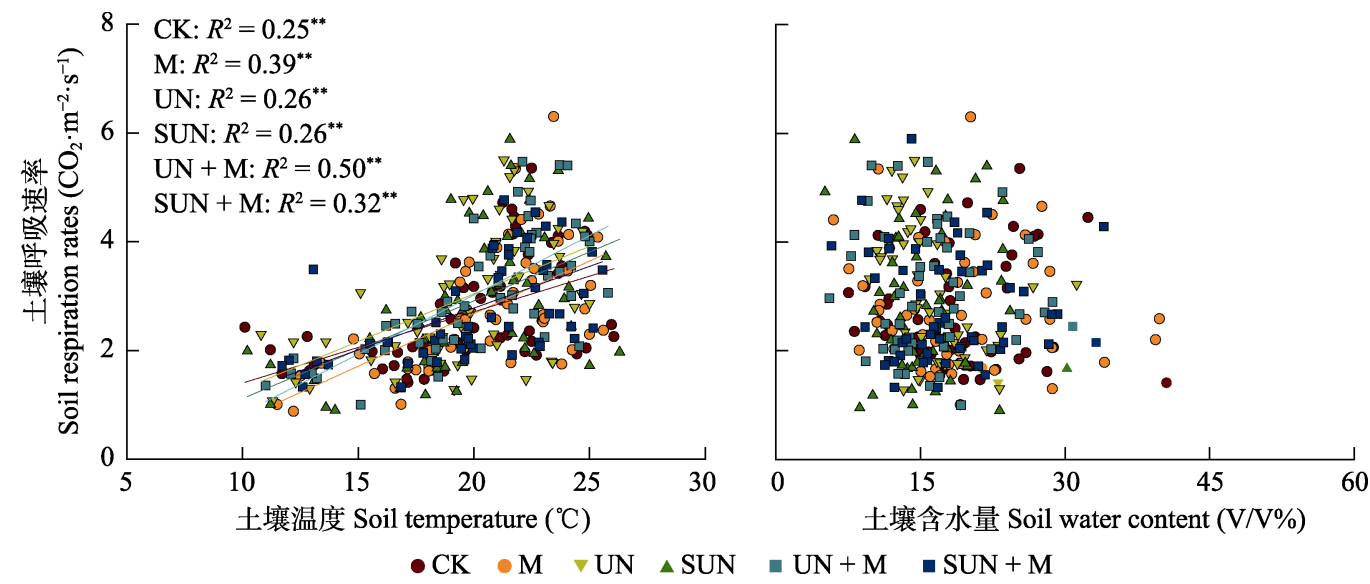

图5 北方农牧交错带温性盐碱化草地不同处理的土壤呼吸速率与土壤温度和土壤水分的相关性。CK, 对照处理; M, 刈割 处理; SUN, 添加缓释尿素处理; SUN + M, 添加缓释尿素+刈割处理; UN, 添加尿素处理; UN + M, 添加尿素+刚割处理。 **，相关性在 0.01 水平上显著。

Fig. 5 Correlation between soil respiration rates and soil temperature and moisture under different treatments of saline-alkali grassland in the northern agricultural-pastoral ecotone. CK, control treatment; M, mowing treatment; SUN, slow release urea treatment; SUN + M, slow release urea and mowing treatment; $\mathrm{UN}$, urea treatment; $\mathrm{UN}+\mathrm{M}$, urea and mowing treatment. **, correlation is significant at the 0.01 level.

表3＼cjkstart北方农牧交错带温性盐碱化草地土壤呼吸与土壤微生物，土壤理化性质的相关性

Table 3 Pearson's correlation between soil respiration and soil microorganisms, and soil physical and chemical properties of saline-alkaline grasslands in the northern agricultural-pastoral ecotone

\begin{tabular}{|c|c|c|c|c|c|c|c|c|c|c|c|}
\hline & SR & $\mathrm{NH}_{4}^{+}$ & $\mathrm{NO}_{3}^{-}$ & BNPP & AGB & BGB & Litter & $\mathrm{MBC}$ & MBN & $\mathrm{MBC} / \mathrm{MBN}$ & DOC \\
\hline SR & 1.000 & & & & & & & & & & \\
\hline $\mathrm{NH}_{4}^{+}$ & $-0.412^{* *}$ & 1.000 & & & & & & & & & \\
\hline $\mathrm{NO}_{3}^{-}$ & $-0.321^{* *}$ & $0.450^{* *}$ & 1.000 & & & & & & & & \\
\hline BNPP & 0.072 & 0.108 & $0.295^{*}$ & 1.000 & & & & & & & \\
\hline AGB & -0.013 & $0.468^{* *}$ & $0.433^{* *}$ & $0.233^{*}$ & 1.000 & & & & & & \\
\hline BGB & 0.005 & -0.139 & $-0.358^{* *}$ & -0.102 & -0.032 & 1.000 & & & & & \\
\hline Litter & -0.189 & $0.622^{* *}$ & $0.536^{* *}$ & 0.180 & $0.489^{* *}$ & -0.193 & 1.000 & & & & \\
\hline MBC & $0.253^{*}$ & $-0.363^{* *}$ & $-0.372^{* *}$ & -0.029 & -0.138 & $0.380^{* *}$ & $-0.337^{* *}$ & 1.000 & & & \\
\hline MBN & 0.191 & $-0.314^{* *}$ & $-0.248^{*}$ & -0.231 & -0.219 & $0.316^{* *}$ & $-0.281^{*}$ & $0.561^{* *}$ & 1.000 & & \\
\hline $\mathrm{MBC} / \mathrm{MBN}$ & 0.026 & 0.166 & -0.066 & 0.215 & 0.043 & 0.162 & 0.026 & 0.185 & $-0.306^{* *}$ & 1.000 & \\
\hline DOC & 0.194 & -0.190 & $-0.410^{* *}$ & -0.180 & -0.134 & 0.187 & $-0.341^{* *}$ & $0.327^{* *}$ & 0.096 & 0.064 & 1.000 \\
\hline
\end{tabular}

*, 相关性在 0.05 水平上显著; **, 相关性在 0.01 水平上显著。 AGB, 地上生物量; BGB, 地下生物量; BNPP, 净地下生物量; DOC, 可溶性有机碳; Litter, 调落物生物量; MBC, 微生物生物量碳; MBN, 微生物生物量氮; SR, 土壤呼吸速率。

*, correlation is significant at the 0.05 level; **, correlation is significant at the 0.01 level. BNPP, net below-ground biomass; AGB, above-ground biomass; $\mathrm{BGB}$, below-ground biomass; Litter, litter biomass; MBC, microbial biomass carbon; MBN, microbial biomass nitrogen; DOC, dissolved organic carbon; $\mathrm{SR}$, soil respiration rates.

www.plant-ecology.com 


\section{2 土壤呼吸对刏割的响应}

我们的研究发现刈割处理显著降低土壤 $\mathrm{CO}_{2}$ 排 放量 $(p<0.05)$, 表明刚割是草地有效的碳管理方 式。刚割导致土壤碳排放降低可能是由于刈割减少 了土壤的植被覆盖度和枯落物的积累, 使得植物累 积和输入到土壤中的养分较少, 减少了土壤微生物 呼吸的底物(康颖和侯扶江, 2011; 宗宁等, 2013; Zhang et al., 2017b), 致使根系和土壤微生物呼吸作 用减弱(孙振中等, 2012), 从而减少了碳排放。同时 刈割会增加土壤温度, 降低土壤含水量。土壤温度 是调节土壤呼吸的重要因素, 它不仅直接影响自养 呼吸和异养呼吸过程, 还会通过影响根和微生物生 物量间接调节土壤呼吸(Peng et al., 2017)。土壤含水 量的多少影响根系生长、根系呼吸、土壤微生物群 落构成、微生物活力以及代谢活力, 进而影响土壤 呼吸(郭明英等, 2011，2012)。有研究发现每15天进 行一次刈割处理会使土壤碳排放量成倍增长(马俐 等, 2016), 而本研究发现每个生长季进行一次刈割 处理会降低土壤碳排放, 这说明与高频次刈割(过 度刈割)相比, 低频次刈割(适度刈割)有利于减少土 壤 $\mathrm{CO}_{2}$ 排放量, 降低农牧交错带盐碱化草地土壤碳 源作用。

氮添加和则割的短期实验表明，二者的交互作 用对土壤呼吸速率没有显著影响。氮添加和刚割短 期内对地上植被物种组成和土壤微生物群落结构没 有影响, Du等(2018)在北方温带草地进行 10 年的氮 添加和刈割研究也发现, 氮添加和刈割的交互作用 对土壤呼吸无显著影响。但是氮添加导致的地上生 物量的增加能否抵消刈割带走大量生物量导致的碳 输入降低, 氮添加和刈割的交互作用对草地土壤呼 吸的影响及其机理还有待深入研究。

\section{4 结论}

短期氮添加显著提高了北方农牧交错带盐碱化 草地土壤呼吸速率, 短期刚割处理显著降低土壤呼 吸速率, 氮添加和刈割之间没有显著交互作用。土 壤呼吸速率对氮添加和刈割的响应受到氮添加累积 量和刈割处理年限的影响。另外, 氮添加提高土壤 呼吸的同时提高了初级生产力, 而刈割降低土壤呼 吸的同时会减少土壤有机碳的输入, 随着氮添加和 刏割处理时间的延长, 氮添加是否能抵消刏割导致 的碳输入减少, 长期施氮和刏割条件下, 农牧交错
带盐碱化草地土壤的碳源汇问题有待进一步进行 长期深入地研究。

\section{参考文献}

Chen DM, Li JJ, Lan ZC, Hu SJ, Bai YF (2016). Soil acidification exerts a greater control on soil respiration than soil nitrogen availability in grasslands subjected to long-term nitrogen enrichment. Functional Ecology, 30, 658-669.

Chen DM, Wang TJ, Yu SJ, Jin Y (2002). Review on the research and development of control-release urea and slow-release urea. Chemical Industry and Engineering Progress, 21, 455-461. [陈德明, 王亭杰, 雨山江, 金涌 (2002). 缓释和控释尿素的研究与开发综述. 化工进展, 21, 455-461.]

Du Y, Han HY, Wang YF, Zhong MX, Hui DF, Niu SL, Wan SQ (2018). Plant functional groups regulate soil respiration responses to nitrogen addition and mowing over a decade. Functional Ecology, 32, 1117-1127.

Guo MY, Chao KT, You JC, Xu LJ, Wang LJ, Jia SJ, Xin XP (2012). Soil microbial characteristic and soil respiration in grassland under different use patterns. Acta Agrestia sinica，20，42-48. [郭明英, 朝克图, 尤金成, 徐丽君, 王丽娟，贾淑杰，辛晓平 (2012). 不同利用方式下草地 土壤微生物及土壤呼吸特性. 草地学报, 20, 42-48.]

Guo MY, Wei ZJ, Xu LJ, Yang GX, Liu HM, Wu YL, Xin XP (2011). Soil respiration of different mowing types of meadows. Acta Agrestia Sinica, 19, 51-57. [郭明英, 卫智 军, 徐丽君, 杨桂霞, 刘红梅, 吴艳玲, 辛晓平 (2011). 不同刈割年限天然草地土壤呼吸特性研究. 草地学报, 19, 51-57.]

He LH, Dong GH, Wang WM, Ming Z (2014). Ecosystem status and change assessment of Agro-Pastoral Ecotone of North China in 2000-2010. Environmental Monitoring in China，30(5)，63-68. [何立环，董贵华，王伟民，明珠 (2014). 中国北方农牧交错带2000-2010年生态环境状 况分析. 中国环境监测, 30(5), 63-68.]

He YL, Qi YC, Peng Q, Dong YS, Yan ZQ, Li ZL (2018). Effects of exogenous carbon and nitrogen addition on the key process of carbon cycle in grassland ecosystem: A review. China Environmental Science, 38, 1133-1141. [贺云龙, 齐玉春, 彭琴, 董云社, 问钟清, 李兆林 (2018). 外源 碳氮添加对草地碳循环关键过程的影响. 中国环境科 学, 38, 1133-1141.]

Hu W, Zhang YH, Li P, Zhang P, Li MY, You JT, Tian SQ (2019). Effects of different levels of nitrogen fertilization on soil respiration rates and soil biochemical properties in an alfalfa grassland. Environmental Science, 40, 28582868. [胡伟, 张亚红, 李鹏, 张鹏, 李满友, 尤璟涛, 田 水泉 (2019). 不同施氮水平对紫花苜宿草地土壤呼吸 和土壤生化性质的影响. 环境科学, 40, 2858-2868.]

Kang HZ, Fahey TJ, Bae K, Fisk M, Sherman RE, Yanai RD, 
See CR (2016). Response of forest soil respiration to nutrient addition depends on site fertility. Biogeochemistry, 127, 113-124.

Kang J, Ren HY, Wang YH, Han MQ, Jin YX, Yan BL, Han GD (2019). Responses of soil respiration to long-term climate warming and nitrogen fertilization in a desert steppe. Journal of Arid Land Resources and Environment, 33(5), 151-157. [康静, 任海燕, 王悦骅, 韩梦琪, 靳宇曦, 问 宝龙, 韩国栋 (2019). 短花针茅荒漠草原土壤呼吸对长 期增温和氮素添加的响应. 干旱区资源与环境, 33(5), 151-157.]

Kang Y, Hou FJ (2011). Response of soil respiration rate of alfalfa grassland to cutting treatment. Pratacultural Science, 28, 892-897. [康颖, 侯扶江 (2011). 黄土高原紫 花苜宿草地土壤呼吸对刈割的响应. 草业科学, 28 , 892-897.]

Lal R (2004). Soil carbon sequestration impacts on global climate change and food security. Science, 304, 1623-1627.

Li B (1997). The rangeland degradation in north China and its preventive strategy. Scientia Agricultura Sinica, 30(6), 1-9. [李博 (1997). 中国北方草地退化及其防治对策. 中国农业科学, 30(6), 1-9.]

Li CB, Peng YF, Nie XQ, Yang YH, Yang LC, Li F, Fang K, Xiao YM, Zhou GY (2018a). Differential responses of heterotrophic and autotrophic respiration to nitrogen addition and precipitation changes in a Tibetan alpine steppe. Scientific Reports, 8, 16546. DOI: 10.1038/s41598-01834969-5.

Li JJ, Huang Y, Xu FW, Wu LJ, Chen DM, Bai YF (2018b). Responses of growing-season soil respiration to water and nitrogen addition as affected by grazing intensity. Functional Ecology, 32, 1890-1901.

Liu LL, Greaver TL (2010). A global perspective on belowground carbon dynamics under nitrogen enrichment. Ecology Letters, 13, 819-828.

Ma L, Zhou ZQ, Wang ZW (2016). Effects of mowing and nitrogen addition on carbon sequestration of Leymus chinensis grasslands in the Songnen Plain, Northeast China. Chinese Journal of Ecology, 35, 87-94. [马俐, 周 志强, 王正文 (2016). 刈割和氮添加对松嫩平原羊草草 原碳固持的影响. 生态学杂志, 35, 87-94.]

Mi YB, Yang JS, Yao RJ, Yu SP (2016). Effects of farming practice on soil respiration, $\mathrm{EC}_{\mathrm{e}}$ and organic carbon in coastal saline soil. Acta Pedologica Sinica, 53, 612-620. [米迎宾, 杨劲松, 姚荣江, 余世鹏 (2016). 不同措施对 滨海盐渍土壤呼吸、电导率和有机碳的影响. 土壤学报, 53, 612-620.]

Peng Q, Dong YS, Qi YC, Xiao SS, He YT, Ma T (2011). Effects of nitrogen fertilization on soil respiration in temperate grassland in Inner Mongolia, China. Environmental Earth Sciences, 62, 1163-1171.

Peng YF, Li F, Zhou GY, Fang K, Zhang DY, Li CB, Yang
GB, Wang GQ, Wang J, Mohammat A, Yang YH (2017). Nonlinear response of soil respiration to increasing nitrogen additions in a Tibetan alpine steppe. Environmental Research Letters, 12, 024018. DOI: 10.1088/1748-9326/ aa5ba6.

Qi YC, Liu XC, Dong YS, Peng Q, He YT, Sun LJ, Jia JQ, Cao CC (2014). Differential responses of short-term soil respiration dynamics to the experimental addition of nitrogen and water in the temperate semi-arid steppe of Inner Mongolia, China. Journal of Environmental Sciences, 26, 834-845.

Schlesinger WH, Andrews JA (2000). Soil respiration and the global carbon cycle. Biogeochemistry, 48, 7-20.

Sun HY, Zhao JP, Xiao YL, Feng JW, Zhang ZG (2018). Soil respiration and its response to simulated nitrogen deposition in a subtropical evergreen broad-leaved forest in Mount Wuyi. Ecology and Environmental Sciences, 27, 1632-1638. [孙海燕, 赵俊平, 肖艳玲, 冯建伟, 张占贵 (2018). 模拟氮沉降对武夷山亚热带常绿阔叶林土壤呼 吸的影响. 生态环境学报, 27, 1632-1638.]

Sun ZZ, Wang JS, Pan GY, Ouyang Z, Li FD, Cheng WX (2012). Effects of cutting on soil respiration rate of cultivated grassland in North China Plain. Journal of Natural Resources, 27, 809-819. [孙振中, 王吉顺, 潘国艳, 欧 阳竹, 李发东, 程维新 (2012). 刈割对华北平原人工草 地土壤呼吸速率的影响. 自然资源学报, 27, 809-819.]

Vance ED, Brookes PC, Jenkinson DS (1987). An extraction method for measuring soil microbial biomass C. Soil Biology \& Biochemistry, 19, 703-707.

Wang D, Chen YJ (2015). Research status of different grassland soil respiration types in China. Journal of Anhui Agricultural Sciences, 43, 74-76, 82. [王丹, 陈永金 (2015). 中国不同类型草地土壤呼吸研究现状. 安徽农业科学, 43, 74-76, 82.]

Wang LF, Zhang PY, Li H, Liu SW (2018). Vulnerability of social-ecosystem in agro-pastoral ecotone in western Northeast China. Journal of University of Chinese Academy of Sciences, 35, 345-352. [王林峰, 张平宇, 李鹤, 刘世薇 (2018). 东北西部农牧交错带社会生态系统脆 弱性. 中国科学院大学学报, 35, 345-352.]

Wang XY, Li YL, Zhao XY, Mao W, Cui D, Qu H, Lian J, Luo YQ (2012). Responses of soil respiration to different environment factors in semi-arid and arid areas. Acta Ecologica Sinica, 32, 4890-4901. [王新源, 李玉霖, 赵学 勇, 毛伟, 崔夺, 曲浩, 连杰, 罗永清 (2012). 干旱半干 旱区不同环境因素对土壤呼吸影响研究进展. 生态学 报, 32, 4890-4901.]

Wei L, Su JS, Jing GH, Zhao J, Liu J, Cheng JM, Jin JW (2018). Nitrogen addition decreased soil respiration and its components in a long-term fenced grassland on the Loess Plateau. Journal of Arid Environments, 152, 37-44.

Xie JX, Zhai CX, Li Y (2008). A comparative study on soil

www.plant-ecology.com 
$\mathrm{CO}_{2}$ flux between a saline desert and a cropped-oasis farmland. Progress in Natural Science, 18, 262-268. [谢 静霞, 翟翠霞, 李彦 (2008). 盐生荒漠与绿洲农田土壤 $\mathrm{CO}_{2}$ 通量的对比研究. 自然科学进展, 18, 262-268.]

Ye CL, Chen D, Hall SJ, Pan S, Yan XB, Bai TS, Guo H, Zhang Y, Bai YF, Hu SJ (2018). Reconciling multiple impacts of nitrogen enrichment on soil carbon: Plant, microbial and geochemical controls. Ecology Letters, 21, 1162-1173.

Zhang JJ, Jing XY, de Lajudie P, Ma C, He PX, Singh RP, Chen WF, Wang ET (2016). Association of white clover (Trifolium repens L.) with rhizobia of sv. trifolii belonging to three genomic species in alkaline soils in North and East China. Plant and Soil, 407, 417-427.

Zhang JY, Ai ZM, Liang CT, Wang GL, Xue S (2017a). Response of soil microbial communities and nitrogen thresholds of Bothriochloa ischaemum to short-term nitrogen addition on the Loess Plateau. Geoderma, 308, 112-119.

Zhang Q, Li JX, Xie ZQ (2017). Effects of nitrogen addition on soil respiration of Rhododendron simsii shrubland in the subtropical mountainous areas of China. Chinese Journal of Plant Ecology, 41, 95-104. [张蓄, 李家湘, 谢宗强 (2017). 氮添加对亚热带山地杜鹃灌从土壤呼吸的影响. 植物生态学报, 41, 95-104.]

Zhang YH, Loreau M, He NP, Zhang GM, Han XG (2017b). Mowing exacerbates the loss of ecosystem stability under nitrogen enrichment in a temperate grassland. Functional Ecology, 31, 1637-1646.

Zhang YJ, Yang GW, Liu N, Chang SJ, Wang XY (2013). Review of grassland management practices for carbon sequestration. Acta Prataculturae Sinica, 22, 290-299. [张 英俊, 杨高文, 刘楠, 常书娟, 王晓亚 (2013). 草原碳 汇管理对策. 草业学报, 22, 290-299.]

Zhao TQ, Ouyang ZY, Jia LQ, Zheng H (2004). Ecosystem services and their valuation of China grassland. Acta Ecologica Sinica, 24, 1101-1110. [赵同谦, 欧阳志云, 贾 良清, 郑华 (2004). 中国草地生态系统服务功能间接价 值评价. 生态学报, 24, 1101-1110.]

Zhou ZH, Wang CK, Zheng MH, Jiang LF, Luo YQ (2017). Patterns and mechanisms of responses by soil microbial communities to nitrogen addition. Soil Biology \& Biochemistry, 115, 433-441.

Zong N, Shi PL, Jiang J, Xiong DP, Meng FS, Song MH, Zhang XZ, Shen ZX (2013). Interactive effects of short-term nitrogen enrichment and simulated grazing on ecosystem respiration in an alpine meadow on the Tibetan Plateau. Acta Ecologica Sinica, 33, 6191-6201. [宗宁, 石 培礼, 蒋婧, 熊定鹏, 孟丰收, 宋明华, 张宪洲, 沈振西 (2013). 短期氮素添加和模拟放牧对青藏高原高寒草甸 生态系统呼吸的影响. 生态学报, 33, 6191-6201.]

责任编委: 程晓莉 编辑: 赵 航 